\title{
A non-invasive method for measuring preimplantation embryo physiology
}

\author{
James R. Trimarchi ${ }^{1}$, Lin Liu ${ }^{1,3}$, D. Marshal Porterfield ${ }^{2}$, Peter J.S. Smith ${ }^{2}$ and David L. Keefe ${ }^{1,3}$ \\ Marine Biological Laboratory, Woods Hole, Massachusetts, and Women and Infants Hospital, Providence, \\ Rhode Island, USA
}

Date submitted: 17.9.99. Date accepted: 30.11 .99

\section{Summary}

The physiology of the early embryo may be indicative of embryo vitality and therefore methods for noninvasively monitoring physiological parameters from embryos could improve preimplantation diagnoses. The self-referencing electrophysiological technique is capable of non-invasive measurement of the physiology of individual cells by monitoring the movement of ions and molecules between the cell and the surrounding media. Here we use this technique to monitor gradients of calcium, potassium, oxygen and hydrogen peroxide around individual mouse preimplantation embryos. The calcium-sensitive electrode in self-referencing mode identified a region of elevated calcium concentration $(\sim 0.25$ pmol $)$ surrounding each embryo. The calcium gradient surrounding embryos was relatively steep, such that the region of elevated calcium extended into the medium only $4 \mu \mathrm{m}$ from the embryo. By contrast, using an oxygen-sensitive electrode an extensive gradient of reduced dissolved oxygen concentration was measured surrounding the embryo and extended tens of micrometres into the medium. A gradient of neither potassium nor hydrogen peroxide was observed around unperturbed embryos. We also demonstrate that monitoring the physiology of embryos using the self-referencing technique does not compromise their subsequent development. Blastocysts studied with the self-referencing technique implanted and developed to term at the same frequency as did unexamined, control embryos. Therefore, the self-referencing electrode provides a valuable non-invasive technique for studying the physiology and pathophysiology of individual embryos without hindering their subsequent development.

Keywords: Calcium, Embryo physiology, Embryo transfer, Oxygen, Preimplantation diagnosis

\section{Introduction}

Embryos vary in developmental potential and therefore methods of preimplantation embryo assessment are used in both clinical and agricultural settings to enhance the efficiency of reproductive technologies (Gardner \& Schoolcraft, 1998; Menezo \& Dale, 1998;

All correspondence to: David Keefe, Laboratory of Reproductive Medicine, Lillie Building, Marine Biological Laboratory, 7 MBL Street, Woods Hole, MA 02543, USA. Tel: +1 (508) 289 7640. Fax: +1 (508) 540 6902. e-mail: dkeefe@wihri.org

${ }^{1}$ Laboratory of Reproductive Medicine, Marine Biological Laboratory, Woods Hole, Massachusetts, USA.

${ }^{2}$ BioCurrents Research Center, Marine Biological Laboratory, Woods Hole, Massachusetts, USA.

${ }^{3}$ Women and Infants Hospital, Brown University, Providence, Rhode Island, USA
Veiga et al., 1998). Two general approaches for embryo evaluation are presently being employed: morphological analysis (Giorgetti et al., 1995; Grillo et al., 1991; Ziebe et al., 1997) and molecular genetic diagnosis (Muggleton-Harris et al., 1995; Fasouliotis \& Schenker, 1998; Ray et al., 1998). These techniques, like all techniques, have their limitations. Assays of embryo physiology offer an alternative approach for preimplantation embryo assessment that could complement these existing methods. Several physiological parameters such as nutrient uptake (Leese \& Barton, 1984; Gardner \& Leese, 1987, 1993; Gardner et al., 1989, 1996; Lane \& Gardner, 1996), ion homeostasis (Stachecki \& Armant, 1996; Lane et al., 1998; Lane and Bavister, 1998) or oxygen metabolism (Fridhandler et al., 1957; Mills \& Brinster, 1967; Houghton et al., 1996) have been measured from mammalian embryos; however, the physiological techniques employed to obtain these measurements are either destructive, require indicator dyes, are 
technically challenging or are unsuitable for noninvasive examination of individual embryos (Mitani, 1985; Gardner \& Leese, 1987, 1993; Johnson et al., 1991; Lane \& Gardner, 1996; Houghton et al., 1996; Phillips et al., 1998).

The self-referencing physiological technique offers a unique approach for assaying physiological parameters from individual preimplantation embryos (Smith et al., 1994; Keefe et al., 1995) (Fig. 1). This technique can monitor the physiology of individual cells and embryos by measuring extracellular gradients of ions, oxygen or metabolic byproducts in the medium immediately surrounding the cell (Smith, 1995; Keefe et al., 1995; Porterfield et al., 1998; Land et al., 1999). The exquisite sensitivity of this physiological method arises from reducing the impact of electronic drift by oscillating a single electrode in a square wave between two points 5 or $10 \mu \mathrm{m}$ apart (Fig. 1). One pole of the oscillation measures the physiological signal near the cell without touching the cell. The other pole of the oscillation, several micrometres away from the cell, measures the physiological signal at a position remote from the cell. By obtaining the difference between the measurements at these two sites, the contribution of electronic drift is reduced and the resultant signal obtained is proportional to the difference in the composition of the media away from and immediately surrounding the cell (Fig. 1). This technique has been used for the non-invasive monitoring of physiological parameters from a variety of cell types, including neuronal tissue and epithelial cells (Shirihai et al., 1998; Brenton et al., 1998), and it has recently been employed to monitor the physiology of oocytes (Hill et al., 1999; Pepperell et al., 1999) and embryos (Keefe et al., 1995; Porterfield et al., 1998).

We sought to explore the potential of the self-referencing technique for measuring a variety of physiological parameters from embryos and also determining whether measuring the physiology of embryos using this technique affected their subsequent development. We found that gradients of calcium ions and oxygen surround embryos and that the development of embryos was not perturbed by physiological examination using the self-referencing technique. Therefore, this non-invasive physiological technique is uniquely suited for investigating the physiology and pathophysiology of preimplantation embryos.
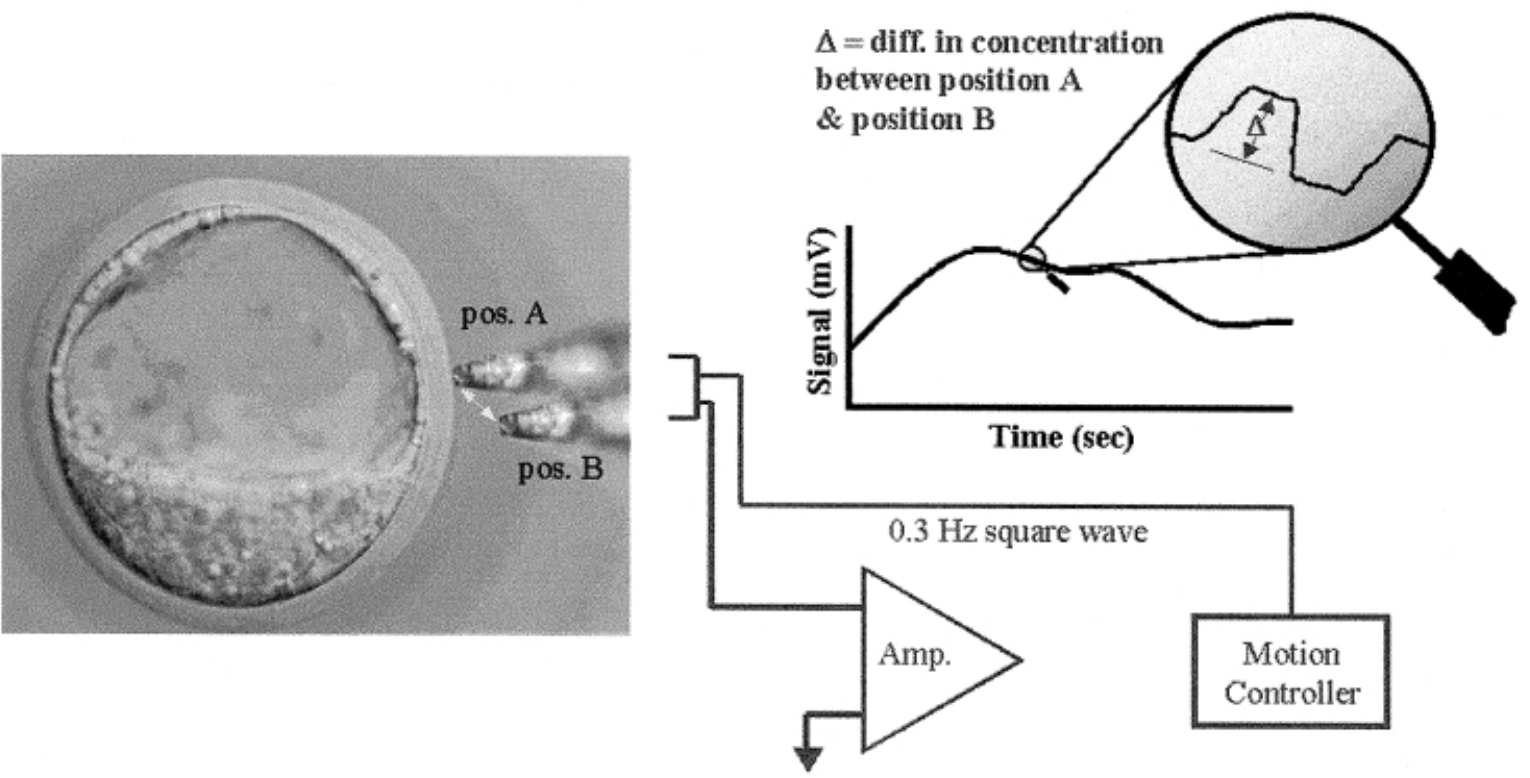

Figure 1 The self-referencing technique employed to measure the physiology of embryos. A digitally generated double exposure of an ion-selective electrode oscillated near a blastocyst in a square wave $(0.3 \mathrm{~Hz})$ between two points 5 or $10 \mu \mathrm{m}$ apart. One pole of the oscillation (pos. A) is near the embryo and measured the physiological signal without touching the embryo. The other pole of the oscillation (pos. B) is several micrometres away from the embryo. The movements are coordinated by a computer-coordinated motion controller. The signal from the electrode is amplified and the difference between the measurements at these two sites $(\Delta)$ is proportional to difference in the concentration of the target molecule between the two positions. Because a single electrode is used the electronic drift (inset graph) is common to both positions and the difference in signal strength at each position can be extracted by frequency- and phase-dependent filtering. The recurrent path for the current is a silver/silver chloride ground electrode in the bath. For further information on the self-referencing technique visit the Nation Center for Research Resources BioCurrents web site at www.mbl.edu/BioCurrents (case sensitive). 


\section{Materials and methods}

We designed and constructed a system for monitoring physiological parameters from embryos using the selfreferencing technique. This system includes a Zeiss Axiovert 100 TV inverted microscope (Carl Zeiss, Thernwood, NY) with a modified stage plate to which computer-controlled micromanipulators are affixed. The microscope rests on a Kinetic Systems Vibraplane vibration-resistant table (Boston, MA) enclosed in a stainless steel insulated chamber. The temperature of the chamber $\left(37^{\circ} \mathrm{C}\right)$ and its contents, including the microscope and manipulators, is controlled by three finned strip heaters regulated by a dual input temperature controller that employs thermocouples positioned throughout the chamber as feedback (parts obtained from Omega Eng., Stamford, CT).

Calcium-sensitive electrodes were fabricated using $\mathrm{Ca}^{2+}$-ionophore I-cocktail A (Fluka, Milwaukee, WI) and backfilled with $100 \mathrm{mM} \mathrm{CaCl}$ as previously decribed (Smith et al., 1999). Potassium-sensitive electrodes were fabricated using $\mathrm{K}^{+}$-ionophore I-cocktail B (Fluka, Milwaukee, WI) and backfilled with $100 \mathrm{mM}$ $\mathrm{KCl}$ as previously described (Shirihai et al., 1998). Oxygen-sensitive electrodes were purchased from Diamond General (Ann Arbor, MI) and used as previously described (Land et al., 1999). Hydrogenperoxide-sensitive electrodes were fabricated by Nafion coating platinum/iridium electrodes purchased from FHC (Bowdoinham, ME). All electrodes had a tip diameter of 3-8 $\mu \mathrm{m}$. All electrodes were calibrated and all ion-selective electrodes were confirmed to be Nerstian prior to use. A silver/silver chloride reference electrode completed the circuit in solution by way of a $3 \mathrm{~mol} \mathrm{l}^{-1} \mathrm{KCl} / 3 \%$ agar bridge. For $\mathrm{K}^{+}$recordings a $3 \mathrm{~mol} \mathrm{l}^{-1} \mathrm{NaCl} / 3 \%$ agar bridge was used. The recording electrode was positioned near the embryo under the control of a computer-guided micromanipulator that allowed movements in three axes with a step size of less than $0.25 \mu \mathrm{m}$. During recording, the electrode was oscillated in a square wave parallel to the electrode axis over a distance of $10 \mu \mathrm{m}$ with a frequency of $0.3 \mathrm{~Hz}$. Data acquisition and manipulation were performed as described previously (Smith, 1995; Land et al., 1999). The hardware and software controlling electrode movements, signal amplification and data acquisition were designed and constructed by the BioCurrents Research Center at the Marine Biological Laboratory, Woods Hole, MA (www.mbl.edu/ BioCurrents [case sensitive]).

Physiological measurements were conducted in HEPES-buffered KSOM containing reduced $\mathrm{NaHCO}_{3}$ (4 $\mathrm{mM})$ and elevated HEPES (14 $\mathrm{mM})$, supplemented with non-essential amino acids ( $1 \mathrm{ml}$ of $100 \times$ stock per $100 \mathrm{ml}$ medium), and without protein supplement (bovine serum albumin, BSA). To enhance detection of calcium gradients, medium calcium concentrations were reduced from $1.7 \mathrm{mM}$ to $30-70 \mu \mathrm{M}$ and osmolarity corrected with $\mathrm{MgCl}_{2}$. Otherwise, the components of the medium were in concentrations consistent with the published KSOM recipe (Lawitts \& Biggers, 1993). The concentration of calcium in the medium was unaltered for measurements of potassium, oxygen and hydrogen peroxide gradients. Embryos could be kept in this medium exposed to the atmosphere at $37^{\circ} \mathrm{C}$ for $1.5 \mathrm{~h}$ without compromising their subsequent viability (see below). Recordings were obtained from embryos in plastic Petri dishes with a cover glass bottom coated with poly-L-lysine (MatTek, Ashland, MA) which facilitated embryo positional stabilisation. Typically, a set of 4-6 embryos were placed in a dish and at least three independent recordings were obtained over several min from each embryo in a semi-randomised order. Approximately $60 \mathrm{~min}(56 \pm 14 \mathrm{~min})$ was required to complete the measurements from a set of embryos.

Female B6C3F1 or CD1 mice (6 weeks old) were purchased from Charles River (Boston, MA) and subjected to a $14 \mathrm{~h}: 10 \mathrm{~h}$ light:dark cycle for at least 2 weeks before use. Animals were cared for according to procedures approved by the Marine Biological Laboratory and Women and Infants Hospital Animal Care Committees. Male B6C3F1 or CF1 mice of proven fertility were used for mating. Female mice were superovulated by intraperitoneal injection of $7.5 \mathrm{IU}$ pregnant male serum gonadotrophin (PMSG; Calbiochem, La Jolla, CA) followed 46-48 h later by injection of 7.5 IU human chorionic gonadotrophin (hCG), and mated individually with males. Females exhibiting mating plugs were selected the next morning and killed by cervical dislocation at 22-23 h after hCG injection. Zygotes ( $\sim 30$ per animal) enclosed in cumulus masses were released from the ampullae into HEPES-buffered KSOM (4 mM $\mathrm{NaHCO}_{3}, 14 \mathrm{mM}$ HEPES, non-essential amino acids ( $1 \mathrm{ml}$ of $100 \times$ stock per $100 \mathrm{ml}$ medium) and $0.1 \%$ BSA) supplemented with $0.03 \%$ hyaluronidase (type IV). Cumulus cells were gently removed from zygotes by pipetting. Cumulus-free zygotes were washed in HEPES-buffered KSOM three times and then in preequilibrated modified KSOM three times. The KSOM used for in vitro culture was supplemented with nonessential amino acids $\mu 1 \mathrm{ml}$ of $100 \times$ stock per $100 \mathrm{ml}$ medium) and 2.5 mM HEPES. Embryos were pooled, randomly distributed and cultured in $50 \mu$ droplets under mineral oil at $37^{\circ} \mathrm{C}$ in a humidified atmosphere of $5 \% \mathrm{CO}_{2}$ in air. These embryo harvest and culture procedures sustained development of control 1-cell zygotes to blastocysts (>90\%) and blastocysts resulting from 4 days of in vitro culture developed to viable offspring when transferred to pseudopregnant female mice. For some experiments blastocysts $(\sim 30$ per animal) were harvested from the uterine horns of female mice at $92-96 \mathrm{~h}$ after hCG injection and mating. 
All other culture and chemical treatment procedures were similar to those employed for zygotes. For some experiments, the zona pellucida was removed mechanically after mild treatment with pronase $(0.5 \%)$. No difference in physiological signals was observed if the embryos were examined immediately after zona removal or after a $0.5-2.5 \mathrm{~h}$ incubation/recovery period. Zona-free embryos cultured individually cleaved and developed to blastocysts in a manner similar to zona-intact sibling embryos when cultured individually. All reagents were purchased from Sigma Chemical Co. (St Louis, MO), unless stated otherwise.

\section{Results}

\section{Calcium gradients}

A region of elevated calcium concentration surrounding individual mouse embryos was monitored reliably using the ion-sensitive self-referencing electrode. The concentration of calcium in the medium immediately surrounding embryos was elevated above that of the bulk medium such that a gradient of calcium emanated in all directions from embryos. Different developmental stages exhibited similar calcium gradients (Fig. 2A). Zona-pellucida-intact (ZP-intact) MII oocytes, 2-cell embryos and blastocysts all exhibited calcium gradients with a maximum calcium concentration near the embryo that was approximately 0.25 pmol above that of the bulk medium or background concentration. Different embryos exhibited markedly different maximum calcium concentrations near the embryo, such that some embryos exhibited calcium signals twice that of other embryos (Fig. 2A). For example, several ZPintact blastocysts concentrated calcium to $>0.8 \mathrm{pmol}$ above that of the bulk medium, while other morphologically indistinguishable embryos exhibited calcium concentrations that were $<0.2 \mathrm{pmol}$ above background (Fig. 2A). We do not know whether these large variations in the magnitude of the maximum calcium signal around different embryos reflect embryo vitality.

Although signals could be measured reliably from ZP-intact embryos, removal of the zona (ZP-free) allowed the electrode to be positioned closer to the plasma membrane and yielded an enhanced signal amplitude (Fig. 2A). Despite the enhanced signal strength from ZP-free embryos, different developmental stages displayed similar elevated calcium concentrations near the embryo. For example, ZP-free 2-cell embryos and blastocysts exhibited similar calcium gradients with a maximum calcium concentration near the plasma membrane that was approximately $1.2 \mathrm{pmol}$ above that of the medium. Perhaps surprisingly, no regional differences in calcium gradients could be detected around blastocysts. Calcium gradients
A
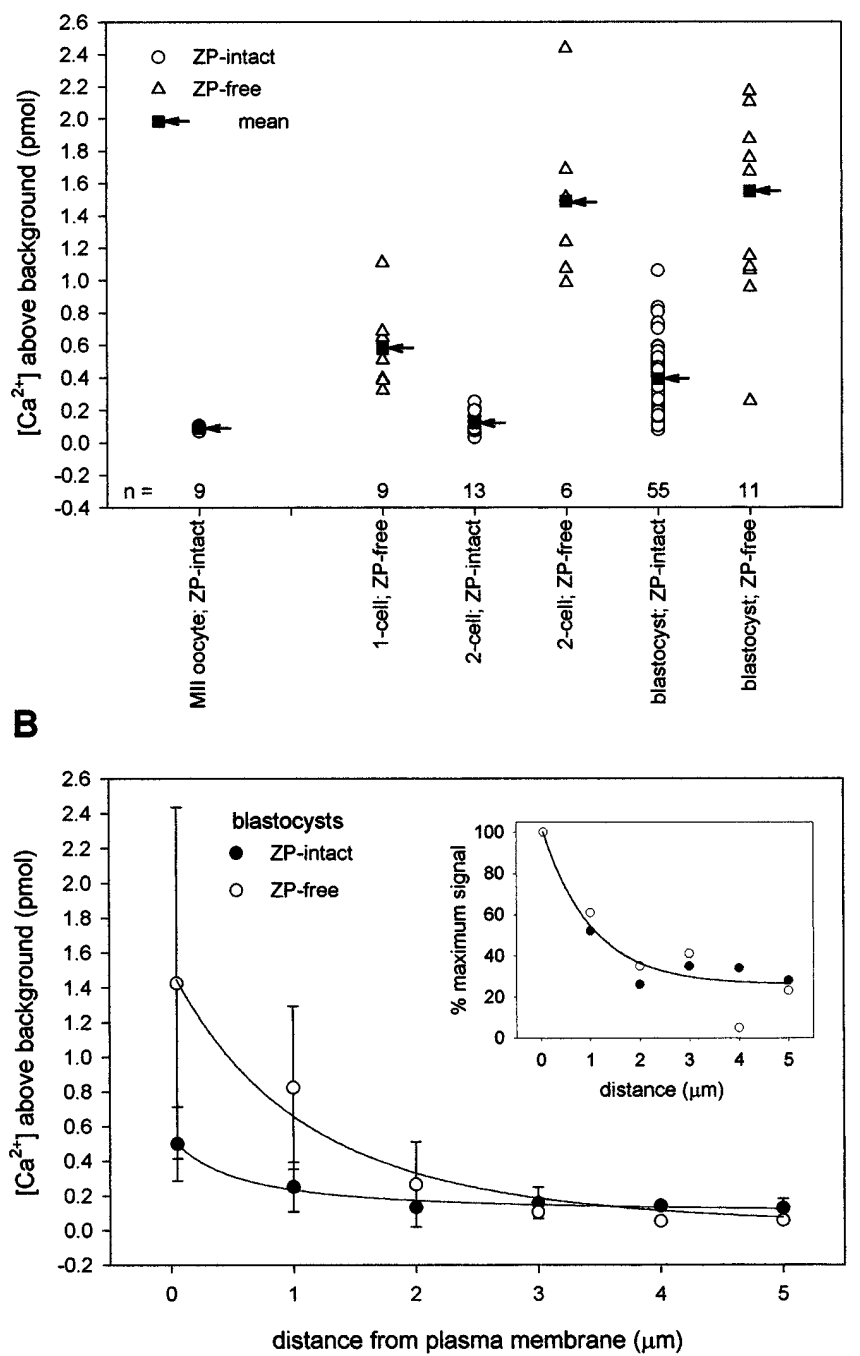

Figure 2 Calcium gradients surrounding preimplantation mouse embryos. (A) The calcium concentration around zona pellucida (ZP)-intact and ZP-free embryos of various developmental stages. Measurements are expressed as the calcium concentration above that in the bulk medium or background concentration (background $=30-70 \mu \mathrm{M}$ calcium and is represented by 0 pmol). Different developmental stages exhibit similar calcium gradients. Within any group different embryos exhibited markedly different calcium gradients. The number of embryos tested in each group is displayed at the bottom of the graph. $(B)$ The calcium gradient profile surrounding blastocysts decreases sharply with increasing distance from the embryo. The calcium concentration profile around 36 of the $55 \mathrm{ZP}$-intact blastocysts compiled for $(A)$ is plotted with filled circles while the open circles represent the gradient profile around 5 of the $11 \mathrm{ZP}$-free blastocysts also shown in $(A)$. The distance of zero ( 0 ) from the embryo is the closest position of the electrode that did not touch the embryo $(<0.5 \mu \mathrm{m})$. Inset: ZP-intact and ZP-free embryos exhibit similar calcium gradient profiles when normalised to the maximum signal obtained nearest the embryo. 
around blastocysts near the inner cell mass (ICM) were similar to those from the opposite side of the blastocyst where the cells are predominantly trophectoderm. We are presently investigating whether the diffusional properties of calcium account for this signal normalisation.

The elevated calcium concentration surrounding each embryo rapidly decreased with increasing distance from the embryo, such that the signal amplitude decreased to only $55 \%$ of its maximum amplitude over the first micrometre away from the embryo, and only $30 \%$ of the signal remained $2 \mu \mathrm{m}$ from the embryo (Fig. $2 B$ ). The elevated calcium concentration observed around embryos decreased to that of the bulk medium by $4 \mu \mathrm{m}$ away from the embryo. Calcium gradients from ZP-intact and ZP-free embryos exhibited similar profiles when normalised to the maximum signal obtained nearest the embryo (Fig. $2 B$, inset). No difference in calcium gradients was observed if the embryos were examined immediately following ZP removal or subsequent to a $1-2.5 \mathrm{~h}$ incubation/recovery period after ZP removal.

\section{Oxygen gradients}

A region of depleted, dissolved oxygen concentration surrounding individual mouse embryos was monitored reliably using the oxygen-sensitive self-referencing electrode (Fig. 3) and was indicative of oxygen consumption by the embryo. The dissolved oxygen concentration of the medium near 1-cell embryos was $0.33 \pm 0.07 \mu \mathrm{mol}$ below the concentration in the bulk medium or background concentration. The maximum magnitude of this oxygen gradient near blastocysts was approximately twice that near 1-cell embryos (Fig. $3 B$ ), suggesting enhanced respiratory metabolism and increased oxygen consumption at the blastocyst stage. These oxygen concentration gradients equate to each blastocyst consuming approximately $11 \mathrm{nmol}$ of oxygen per hour and are consistent with oxygen consumption measurements obtained from groups of embryos (Boell \& Nicholas, 1948; Fridhandler et al., 1957; Mills \& Brinster, 1967; Manes \& Lai, 1995; Houghton et al., 1996). No significant regional differences in oxygen gradients could be detected around blastocysts. Oxygen gradients around blastocysts near the ICM were similar to those from the opposite side of the blastocyst where the cells are predominantly trophectoderm. ZP-intact and ZP-free embryos exhibited similar oxygen gradient maxima.

In contrast to the steep calcium gradients observed around embryos, the oxygen gradients were significantly more shallow and extended tens of micrometres into the medium around the embryos (compare Figs. $2 B$ and $3 A$ ). For example, the oxygen gradient decreased to only $85 \%$ of its maximum amplitude over the first micrometre away from the embryo while the calcium gradient had decreased to $55 \%$ of its maximum amplitude over a similar distance. The oxygen gradient could be measured reliably as far as $20 \mu \mathrm{m}$ away from embryos -4 times the distance at which the calcium gradient had fallen to background levels. Oxygen gradients from ZP-intact and ZP-free embryos exhibited similar profiles.
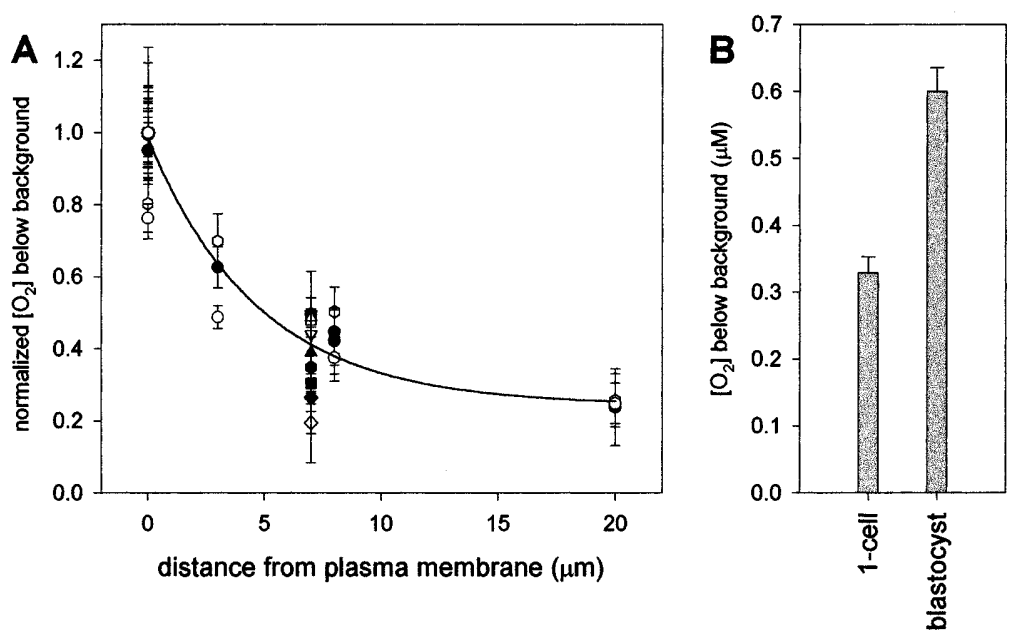

Figure 3 Oxygen gradients surrounding preimplantation mouse embryos. $(A)$ The dissolved oxygen concentration gradient around 1-cell embryos decreases with increasing distance from the embryo. The oxygen gradient decreased to $50 \%$ of its maximum amplitude over the first $5 \mu \mathrm{m}$ away from the embryo. Error bars represent standard deviation $(n=12)$. The distance of zero (0) from the embryo is the closest position of the electrode that did not touch the embryo $(<0.5 \mu \mathrm{m})$. (B) Blastocysts exhibit an oxygen gradient maximum twice as large as do 1-cell embryos. Measurements (mean \pm standard deviation) are expressed as the dissolved oxygen concentration below that in the bulk medium or background concentration (background $=210 \mu \mathrm{M}$ oxygen and is represented by $0 \mu \mathrm{M})$. ( $n=8$ blastocysts and 7 one-cell embryos.) 


\section{Potassium and hydrogen peroxide gradients}

The medium potassium concentration near embryos was similar to that of the bulk medium, in that no potassium gradient was measured surrounding any normal resting embryos. The average potassium concentration near 1-cell embryos $(n=21)$ was $0.02 \pm 0.02$ $\mu \mathrm{M}$ above that of the bulk medium and indistinguishable from background levels. The potassium-sensitive electrodes exhibit a detection limit of $0.01 \mu \mathrm{M}$ at a signal-to-noise ratio of 3. Similarly, the hydrogen peroxide concentration near 1-cell embryos or blastocysts did not differ appreciably from that of the bulk medium. The average hydrogen peroxide concentration near 1-cell embryos $(n=8)$ and blastocysts $(n=10)$ was $0.015 \pm 0.039 \mu \mathrm{M}$ and $0.022 \pm 0.025 \mu \mathrm{M}$, respectively, and was indistinguishable from background levels. The hydrogen peroxide-sensitive electrodes exhibit a detection limit of $0.01 \mu \mathrm{M}$ at a signal-to-noise ratio of 3 .

\section{The self-referencing electrode does not compromise subsequent embryo development}

To determine whether physiological examination by the self-referencing technique inhibited subsequent development, we measured calcium and oxygen gradients around embryos and monitored their subsequent development. Approximately $60 \mathrm{~min}$ (56 \pm 14 $\mathrm{min}$ ) is required to complete the measurements from a set of 3-5 embryos and embryos could be maintained under the conditions for physiological examination (HEPES-buffered medium, atmospheric gas pressures,
$37{ }^{\circ} \mathrm{C}$ ) for $1.5 \mathrm{~h}$ without compromising their subsequent development. Physiological examination of cleavage stage embryos did not alter their development to blastocysts in vitro. More than $90 \%$ of physiologically examined 1- and 2-cell embryos developed to the blastocyst stage $\left(n=20\right.$ for $\mathrm{Ca}^{+2}$ and $n=30$ for oxygen). A similar percentage of control, unexamined embryos also developed to the blastocyst stage.

The self-referencing technique did not compromise subsequent development to term. Calcium gradients were measured from blastocysts flushed from oviducts and thereafter transferred to pseudopregnant females (Fig. 4, Table 1$)$. Nearly $80 \%(78.1 \pm 10.0 \% ; n=73)$ of blastocysts examined with the self-referencing electrode developed to term after transfer to pseudopregnant hosts. This high degree of development to term occurred despite embryo exposure to the physiological setup outside the incubator for approximately $1 \mathrm{~h}$ (mean $56.3 \pm 13.8 \mathrm{~min}$; max. $88 \mathrm{~min}$ ). Similarly, $80 \%$ $(80.3 \pm 13.9 \% ; n=86)$ of control embryos that had not undergone physiological examination developed to term. The development to term of ZP-free blastocysts was slightly lower than that of ZP-intact blastocysts; however, physiological testing using the self-referencing technique did not inhibit the development of either ZP-intact or ZP-free blastocysts.

\section{Discussion}

The self-referencing electrode technique (Smith, 1995; Land et al., 1999) provides a useful tool for noninvasive measurement of physiological parameters

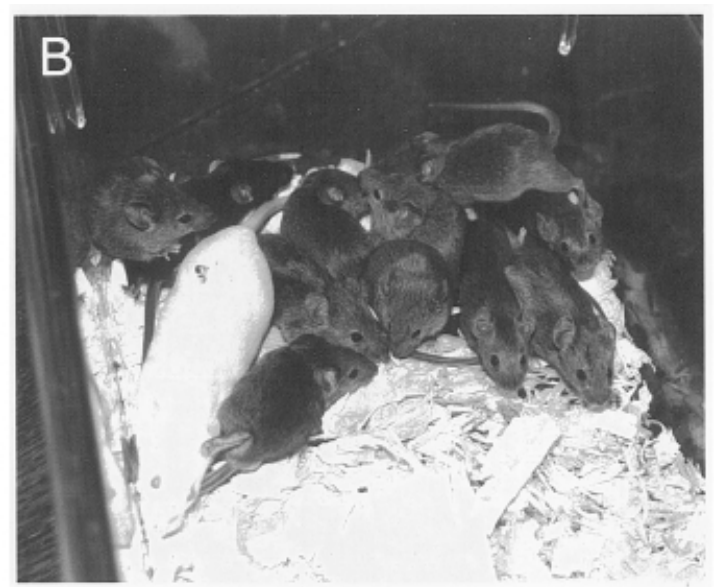

Figure 4 Physiological measurements of blastocysts using the self-referencing electrophysiological technique do not inhibit subsequent development to term. (A) A calcium gradient profile measured near an individual blastocyst using the self-referencing technique (mean \pm standard deviation). Measurements are expressed as the calcium concentration above that in the bulk medium or background concentration (background $=30-70 \mu \mathrm{M}$ calcium and is represented by 0 pmol). Inset: A calcium-sensitive self-referencing electrode positioned near a blastocyst. Although in close proximity to the embryo $(<0.5 \mu \mathrm{m})$, the electrode was not touching the embryo. (B) Aguti (B6C3F1) mice pups born after B6C3F1 blastocysts were physiologically examined and transferred to a white (CD1) pseudopregnant female. One of the mouse pups in $(B)$ most probably resulted from the embryo shown in the inset in $(A)$. See Table 1 for the frequency of development to term. 
Table 1 Survival to term of control and physiologically tested blastocysts

\begin{tabular}{lccc}
\hline$\%$ surviving to term & ZP-intact & ZP-free & Total $^{a}$ \\
\hline Control & $83.9 \pm 14.8 \%$ & $72.9 \pm 12.2 \%$ & $80.3 \pm 13.9 \%$ \\
Total no. of embryos & 48 & 25 & 73 \\
Total no. of pups & 39 & 18 & 57 \\
Trials (recipients) & 4 & $72.5 \pm 10.5 \%$ & 6 \\
& & 37 & $78.1 \pm 10.0 \%$ \\
Physiologically tested & $82.4 \pm 8.9 \%$ & 27 & 86 \\
Total no. of embryos & 49 & 3 & 68 \\
Total no. of pups & 41 & 4 & 7
\end{tabular}

${ }^{a}$ One hundred and fifty-nine blastocysts were transferred into 13 pseudopregnant recipients yielding 125 pups (79\% development to term).

from individual mouse embryos. The goal of this study was to survey a range of physiological parameters that could be monitored from embryos using the self-referencing technique. We have characterised the calcium and oxygen gradients surrounding preimplantation mouse embryos, and have determined that gradients of potassium and hydrogen peroxide are undetectable around unperturbed embryos. We also have demonstrated the non-destructive nature of the self-referencing technique by observing unaltered development to the blastocyst stage in vitro after physiological examination of cleavage stage embryos and by obtaining healthy pups in vivo after physiological examination of blastocysts and transfer to pseudopregnant hosts. We do not know whether the magnitude of physiological signals from different embryos reflects differences in their developmental potential.

\section{Calcium gradients}

We used the self-referencing technique to characterise a region of elevated calcium that surrounds each embryo. It is not surprising that we observed similar calcium gradients around embryos ranging in developmental stage from oocytes to blastocysts. Intracellular calcium concentrations are tightly regulated such that the concentration of calcium within cells is 10000 fold less than that of the medium and calcium homeostasis is critical for early embryo development (Stachecki \& Armant, 1996; Lane et al., 1998; Lane \& Bavister, 1998). Disruptions in calcium homeostasis are known to induce cellular dysfunction and cell death in many cell types (Leist \& Nicotera, 1998; Kroemer et al., 1998) and developmentally incompetent embryos exhibit morphological hallmarks of cell death (Jurisicova et al., 1996; Brison \& Schultz, 1997). Therefore, the self-referencing technique might be used to study the pathophysiology of calcium gradients around embryos in the early stages of cell death.

Although embryos at different developmental stages exhibited similar calcium gradients, within any one developmental stage, different embryos exhibited markedly different calcium gradients. For example, the calcium concentration near some 2-cell embryos was twice that observed surrounding other 2-cell embryos. This greater than 2-fold range in maximum calcium concentration near the embryo may reflect variability in the state of embryos within the particular developmental stage, and indeed calcium plays a role in the cell cycle and cytokinesis (Leung et al., 1998; Ahmed et al., 1998). Alternatively, the variation in calcium gradients we observed between embryos of the same developmental may reflect subtle variations in the physiology of these embryos. The calcium gradients around developmentally competent embryos vary considerably and therefore, at this time, it is unlikely that the magnitude of the maximum calcium signal around different embryos reflects differences in their developmental potential.

The biophysical mechanism underlying the calcium gradients surrounding embryos is presently under investigation. One mechanism that could generate an elevated concentration of calcium around embryos is flux of calcium across the plasma membrane resulting from ATP-dependent calcium pumps and exchangers (Smith, 1995; Pepperell et al., 1999). Once across the plasma membrane, calcium would diffuse into the medium to establish a gradient surrounding embryos similar to that which we observed. Such calcium flux from cumulus-oocyte complexes and mouse ova has been documented (Pepperell et al., 1999; Hill et al., 1999). By contrast, in this study we have characterised the calcium gradient that exists around all embryos continually and most likely does not arise from calcium flux across the plasma membrane alone. The calcium gradient observed around embryos could arise from an attraction of calcium from the bulk medium to the surface charge of the embryo or zona pellucida (Aranda-Espinoza et al., 1999). A deeper understanding of the underlying biophysical 
mechanism generating the calcium gradients described here would help to determine whether these calcium signals might be useful for assessing embryo quality.

\section{Oxygen gradients}

We used the self-referencing technique to characterise a region of decreased dissolved oxygen concentration that surrounds each embryo. By contrast to the steep calcium gradients observed around embryos, the oxygen gradients were significantly more shallow and extended tens of micrometres into the medium around the embryos (compare Figs. $2 B$ and $3 A$ ). For example, the oxygen gradient decreased to only $50 \%$ of its maximum amplitude over the first $5 \mu \mathrm{m}$ away from the embryo while the calcium gradient had decreased to background levels over the same distance. Analysis of the oxygen gradient profile suggests an underlying mechanism of diffusion towards a oxygen sink or consumption of oxygen by the embryos (Trimarchi, in preparation).

We found that blastocysts exhibit oxygen gradients with maxima twice that surrounding zygotes. Others have found a similar increase in oxygen consumption as embryos proceed from the cleavage stages to the blastocyst stage (Boell \& Nicholas, 1948; Fridhandler $e t$ al., 1957; Mills \& Brinster, 1967; Manes \& Lai, 1995; Houghton et al., 1996). In addition, Manes and Lai (1995) found that approximately $25 \%$ of the oxygen utilised by rabbit blastocysts contributes to non-metabolic, surface production of superoxide radicals. Therefore, it is likely that the oxygen gradients we observed around individual mouse embryos result from both metabolic and non-metabolic oxygen consumption.

\section{Potassium and hydrogen peroxide gradients}

Potassium ions play a critical role in establishing the trans-plasma membrane potential that all cells exhibit. Altered potassium homeostasis is characteristic of dying cells (Hughes et al., 1997; McCarthy \& Cotter, 1997) and therefore potassium gradients around embryos may be indicative of poor viability. We found potassium to be in equilibrium across the embryo plasma membrane and no potassium gradient could be measured around healthy embryos. Presently, we are investigating whether embryos exhibiting decreased developmental potential also exhibit altered potassium homeostasis and abnormally generate extracellular potassium gradients that can be detected using the self-referencing technique.

Hydrogen peroxide is a natural by-product of oxidative metabolism and reactive oxygen species detoxification. Cells utilise a variety of mechanisms to eliminate hydrogen peroxide and limit its damaging affects; nevertheless, excess hydrogen peroxide diffuses into the medium surrounding some cells and some cells actively secrete hydrogen peroxide (Briggs et al., 1975; El Khoury et al., 1996). Indeed embryos generate reactive oxygen species (Yang et al., 1998) and hydrogen peroxide has been detected in oocytes, embryos and within the blastocoele (Nasr-Esfahani et al., 1990; Pierce et al., 1991). Healthy blastocysts and 1-cell embryos did not exhibit a gradient of hydrogen peroxide, suggesting that healthy embryos are capable of effectively eliminating hydrogen peroxide using intracellular detoxification mechanisms. Similarly, Manes (1992) demonstrated that blastocysts do not secrete appreciable levels of hydrogen peroxide unless treated with inhibitors for intracellular enzymes that detoxify peroxides.

\section{The self-referencing technique does not inhibit development}

We demonstrate here that the self-referencing technique can monitor physiological parameters from embryos without compromising their viability. Cleavage stage embryos that had been physiologically examined developed to blastocysts at the same frequency as control embryos and in vivo developed blastocysts that were harvested and physiologically examined developed to term at the same frequency as control embryos after transfer to pseudopregnant hosts. These findings verify the non-invasive, non-destructive nature of this exquisitely sensitive physiological technique. The selfreferencing technique can be adapted to monitor a wide range physiological parameters including other ion gradients, $\mathrm{pH}$ gradients, ascorbic acid secretion and nitric oxide production (Smith, 1995; Porterfield, unpublished), and therefore provides a new option for non-invasively investigating the physiology and pathophysiology of the early mammalian embryo.

\section{Acknowledgements}

We thank Richard Sanger and Kasia Hammer for assistance with the self-referencing technique and Gaudenz Danuser for his programming assistance and intellectual support. A portion of this work was funded by an NIH R21 \#RR 12718-02 to D.L.K. and P.J.S.S., KO81099 to D.L.K. and NIH P41 RR01395 to P.J.S.S.

\section{References}

Ahmed, A., Kobayashi, S., Shikasho, T., Nishimura \& Kanaide, H. (1998). Differential effects of $\mathrm{Ca}^{2+}$ transients and cell cycle progression in vascular smooth muscle cells. Eur. J. Pharmacol. 344, 323-31. 
Aranda-Espanoza, H., Chen, Y., Nily, D., Lubensky, T.C., Nelson, P., Ramos, L. \& Weitz, D.A. (1999). Electrostatic repulsion of positively charged vesicles and negatively charged objects. Science 285, 394-7.

Boell, E.J., Nicholas, J.S. (1948). Respiratory metabolism of the mammalian egg. J. Exp. Zool. 109, 267-281.

Brenton, S., Smith, P.J.S., Lui, B. \& Brown, D. (1996). Acidification of the male reproductive tract by proton-pumping ATPase. Nature Med. 2, 470-2.

Briggs, R.T., Drath, D.B., Karnovsky, M.L. \& Karnovsky, M.J. (1975). Localization of NADH oxidase on the surface of human polymorphonuclear leukocytes by a new cytochemical method. J. Cell Biol. 67, 566-86.

Brison, D.R. \& Schultz, R.M. (1997). Apoptosis during mouse blastocyst formation: evidence for a role for survival factors including transforming growth factor $\alpha_{1}$. Biol. Reprod. 56, 1088-96.

El Khoury, J., Hickman, S.E., Thomas, C.A., Cao, L., Silverstein, S.C. \& Loike, J.D. (1996). Scavenger receptor-mediated adhesion of microglia to beta-amyloid fibrils. Nature 382, 716-9.

Fasouliotis, S.J., Schenker, J.G. (1998). Preimplantation genetic diagnosis principles and ethics. Hum. Reprod. 13, 2238-45.

Fridhandler, I., Hafez, E.S.E., Pincus, G. (1957). Developmental changes in the respiratory activity of rabbit ova. Exp. Cell. Res. 13, 132-9.

Gardner, D.K. \& Leese, H.J. (1987). Assessment of embryo viability prior to transfer by the non-invasive measurement of glucose uptake. J. Exp. Zool. 242, 103-5.

Gardner, D.K. \& Leese, H.J. (1993). Assessment of embryo metabolism and viability. In Handbook of In Vitro Fertilization, ed. A. Trounson \& D.K. Gardner, pp. 195-211. Boca Raton: CRC Press.

Gardner, D.K. \& Schoolcraft, W.B. (1998). Human embryo viability: what determines developmental potential and can it be assessed? J. Assist. Reprod. Genet. 15, 455-8.

Gardner, D.K., Clarke, R.N., Lechene, C.P. \& Biggers, J.D. (1989). Development of a noninvasive ultramicrofluorometric method for measuring net uptake of glutamine by single preimplantation embryos. Gamete Res. 24, 427-38.

Gardner, D.K., Pawelczynski, M. \& Trounson, A.O. (1996). Nutrient uptake and utilization can be used to select viable day 7 bovine blastocysts after cryopreservation. Mol. Reprod. Dev. 44, 472-5.

Giorgetti, C., Terriou, P., Auquier, P., Hans, E., Spach, J.L., Salzmann, J. \& Roulier, R. (1995). Embryo score to predict implantation after in-vitro fertilization: based on 957 single embryo transfers. Hum. Reprod. 10, 2427-31.

Grillo, J.M., Gamerre, M., Lacroix, O., Noizet, A. \& Vitry, G. (1991). Influence of the morphological aspect of embryos obtained by in vitro fertilization on their implantation rate. J. In Vitro Fertil. Embryo Transf. 8, 317-21.

Hill, J.L., Hammar, K., Smith, P.J.S. \& Gross, D.J. (1999). Stage dependent effects of epidermal growth factor on $\mathrm{Ca}^{2+}$ efflux in mouse oocytes. Mol. Reprod. Dev. 53, 244-53.

Houghton, F.D., Thompson, J.G., Kennedy, C.J. \& Leese, H.J. (1996). Oxygen consumption and energy metabolism of the early mouse embryo. Mol. Reprod. Dev. 44, 476-85.

Hughes, F.M. Jr, Bortner, C.D., Purdy, G.D., Cidlowski \& J.A. (1997). Intracellular $\mathrm{K}^{+}$suppresses the activation of apop- tosis in lymphocytes. J. Biol. Chem. 272, 30567-76.

Johnson, S.K., Jordan, J.E., Dean, R.G., Page \& R.D. (1991). The quantification of bovine embryo viability using bioluminescent assay for lactate dehydrogenase. Theriogenology 35, 425-33.

Jurisicova, A., Varmuza, S. \& Casper, R.F. (1996). Programmed cell death and human embryo fragmentation. Mol. Hum. Reprod. 2, 93-8.

Keefe, D.L., Pepperell, J., Rinaudo, P., Kunkel, J. \& Smith, P.J.S. (1995). Identification of calcium flux in single preimplantation mouse embryos with the calcium-sensitive vibrating probe. Biol. Bull. 189, 200.

Kroemer, G., Dallaporta, B. \& Resche Rigon, M. (1998). The mitochondria death/life regulator in apoptosis and necrosis. Annu. Rev. Physiol. 60, 619-42.

Land, S.C., Porterfield, D.M., Sanger, R.H. \& Smith, P.J.S. (1999). The self-referencing oxygen-selective microelectrode: detection of transmembrane oxygen flux from single cells. J. Exp. Biol. 202, 211-18.

Lane, M. \& Bavister, B. (1998). Calcium homeostasis in early hamster preimplantation embryos. Biol. Reprod. 59, 1000-7.

Lane, M., Boatman, D.E., Albrecht, R.M. \& Bavister, B.D. (1998). Intracellular divalent cation homeostasis and developmental competence in the hamster preimplantation embryo. Mol. Reprod. Dev. 50, 443-50.

Lane, M. \& Gardner, D.K. (1996). Selection of viable mouse blastocysts prior to transsfer using a metabolic criterion. Hum. Reprod. 11, 1975-8.

Lawitts, J.A. \& Biggers, J.D. (1993). Culture of preimplantation embryos. Methods Enzymol. 225, 153-64.

Leese, H.J. \& Barton, A.M. (1984). Pyruvate and glucose uptake by mouse ova and preimplantation embryos. $J$. Reprod. Fertil. 72, 9-13.

Leist, M. \& Nicotera, P. (1998). Calcium and neuronal death. Rev. Physiol. Biochem. Pharmacol. 132, 79-125.

Leung, C.F., Webb, S.E. \& Miller, A.L. (1998). Calcium transients accompany ooplasmic segregation in zebrafish embryos. Dev. Growth Differ. 40, 313-26.

Manes, C. (1992). Cyanide-resistant reduction of nitroblue tetrazolium and hydrogen peroxide production by the rabbit blastocyst. Mol. Reprod. Dev. 31, 114-21.

McCarthy, J.V. \& Cotter, T.G. (1997). cell shrinkage and appoptosis: a role for potassium and sodium eflux. Cell Death Differ. 4, 756-70.

Manes, C. \& Lai, N.C. (1995). Nonmitochondrial oxygen utilization by rabbit blastocysts and surface production of superoxide radicals. J. Reprod. Fertil. 104, 69-75.

Menezo, Y., Dale, B. (1998). Mammalian embryo quality: is it possible to estimate it, and when? J. Assist. Reprod. Genet. 15, 458-460.

Mills, R.M. \& Brinster, R.L. (1967). Oxygen consumption of preimplantation mouse embryos. Exp. Cell Res. 47, 337-44.

Mitani, S. (1985). The reduction of calcium current associated with early differentiation of the murine embryo. J. Physiol. (Lond.) 363, 71-86.

Muggleton-Harris, A.L., Glazier, A.M., Pickering, S. \& Wall, M. (1995). Genetic diagnosis using polymerase chain reaction and fluorescent in-situ hybridization analysis of biopsied cells from both the cleavage and blastocyst stages of individual cultured human preimplantation embryos. Hum. Reprod. 10, 183-92. 
Nasr-Esfahani, M.H., Aitken, J.R. \& Johnson, M.H. (1990). Hydrogen peroxide levels in mouse oocytes and early cleavage stage embryos developed in vitro or in vivo. Development 190, 501-7.

Pepperell, J.R., Kommineni, K., Buradagunta, S., Smith, P.J.S. \& Keefe, D.L. (1999). Transmembrane regulation of intracellular calcium by a plasma membrane sodium/calcium exchanger in mouse ova. Biol. Reprod. 60, 1137-1143.

Phillips, K.P., Zhou, W.-.I \& Baltz, J.M. (1998). Fluorophore toxicity in mouse eggs and zygotes. Zygote 6, 113-23.

Pierce, G.B., Parchment, R.E. \& Lewellyn, A.L. (1991). Hydrogen peroxide as a mediator of programmed cell death in the blastocyst. Differentiation 46, 181-186.

Porterfield, D.M., Trimarchi, J.R., Keefe, D.L. \& Smith, P.J.S. (1998). Characterization of oxygen and calcium fluxes from early mouse embryos and oocytes. Biol. Bull. 195, 208-9.

Ray, P.F., Ao, A., Taylor, D.M., Winston, R.M. \& Handyside, A.H. (1998). Assessment of the reliability of single blastomere analysis for preimplantation diagnosis of the delta F508 deletion causing cystic fibrosis in clinical practice. Prenat. Diagn. 18; 1402-12.

Shirihai, O., Smith, P., Hammar, K. \& Dagan, D. (1998). Microglia generate external proton and potassium ion gradients utilizing a member of the $\mathrm{H} / \mathrm{K}$ ATPase family. Glia 4, 339-48.
Smith, P.J.S. (1995). The non-invasive probes: tools for measuring transmembrane ion flux. Nature 378, 645-6.

Smith, P.J.S., Sanger, R.H. \& Jaffe, L.F. (1994). The vibrating $\mathrm{Ca}^{2+}$ electrode: a new technique for detecting plasma membrane regions of $\mathrm{Ca}^{2+}$ influx and efflux. Methods Cell Biol. 40, 115-34.

Smith, P.J.S., Hammar, K., Porterfield, D.M., Sanger, R.H. \& Trimarchi, J.R. (1999). A self-referencing, non-invasive, ion-selective electrode for single cell detection of transplasma membrane calcium flux. Microsc. Res. Tech., in press.

Stachecki, J.J., \& Armant, D.R. (1996). Transient release of calcium from inositol 1,4,5-trisphosphate-specific stores regulates mouse preimplantation development. Development 122, 2485-96.

Veiga, A., Boada, M. \& Barri, P.N. (1998). Pre-implantation genetic diagnosis: implications, techniques, and results. Contracept. Fertil. Sex. 26, 568-572.

Yang, H.W., Hwang, K.J., Kwon, H.C., Kwon, H.C., Kim, H.S., Choi, K.W. \& Oh, K.S. (1998). Detection of reactive oxygen species (ROS). and apoptosis in human fragment embryos. Hum. Reprod. 13, 998-1002.

Ziebe, A., Petersen, K., Lindenberg, S., Andersen, A.G., Gabrielsen, A. \& Andersen, A.N. (1997). Embryo morphology or cleavage stage: how to select the best embryos for transfer after in-vitro fertilization. Hum. Reprod. 12, 1545-9. 